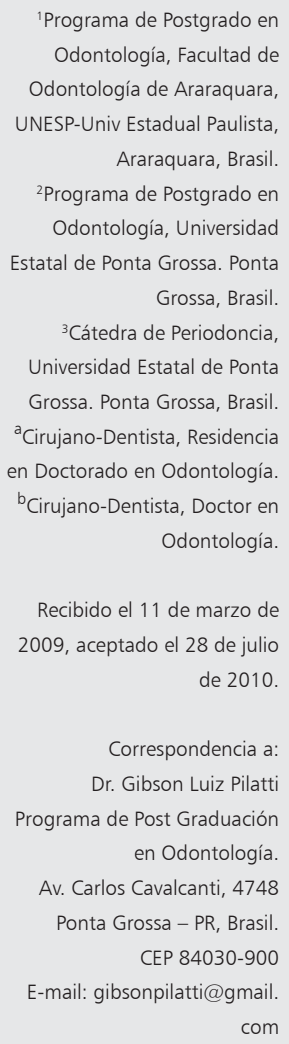

\section{Influencia de la enfermedad periodontal en el control metabólico de pacientes con diabetes mellitus tipo 2: Revisión de la literatura}

\author{
JOÃO PAULO STEFFENS ${ }^{1, \mathrm{a},}$, STELLA MARIA GLACI REINKEE,a, \\ MIGUEL ANGEL MUÑOZ ${ }^{2, a}$, FÁBIO ANDRÉ DOS SANTOS 3 , \\ GIBSON LUIZ PILATTI ${ }^{3, \mathrm{~b}}$
}

\section{Review on periodontal disease and metabolic control of diabetes mellitus}

There may be an interaction between periodontal disease and some systemic diseases such as diabetes mellitus. The objective of this review was to verify, by means of a review of clinical trials, if there is a positive association between periodontal disease and the glycemic control of type 2 diabetes mellitus (DM-2) patients. Eleven articles that fit the study criteria were revised. It was concluded that periodontal disease may influence the metabolic control of DM-2. Additional studies with larger sample sizes and longer follow up are necessary for a better clarification of this issue.

(Rev Med Chile 2010; 138: 1172-1178).

Key words: Blood glucose; Diabetes Mellitus, Type 2; Periodontal diseases.
L a enfermedad periodontal es un proceso patológico que afecta los tejidos del periodonto. El periodonto está constituido por la mucosa masticatoria (periodonto de protección), hueso alveolar, ligamento periodontal y cemento (periodonto de inserción). Esta enfermedad se caracteriza por la inflamación de los tejidos periodontales debido a un proceso infeccioso que puede llevar a una destrucción crónica de estos tejidos, conduciendo a la formación de sacos periodontales (perdidas de inserción) y a la consecuente pérdida dental ${ }^{1}$. El tratamiento de esta enfermedad consiste básicamente en la remoción de cálculos (por medio de destratraje y pulido radicular) y el control del biofilm dental a través de una orientación e instrucción de la higiene bucal y control profesional.

Algunas investigaciones demostraron la influencia de la diabetes mellitus (DM) en la evolución de la enfermedad periodontal ${ }^{2-4}$. Novak et $\mathrm{al}^{5}$, verificaron que pacientes hispanoamericanos con DM-2 presentaban más calculo supra y subgingival, mayor extensión y severidad de destrucción periodontal y una mayor frecuencia de pérdida dental por periodontitis. Una de las razones para esta asociación, es que la DM promueve la liberación de citoquinas proinflamatorias, que modulan la respuesta del huesped y lo tornan más susceptible a la infección periodontal ${ }^{6}$. Además de esto, las altas concentraciones de glucosa sanguínea en pacientes diabéticos llevan a una producción elevada de productos finales de glicosilación avanzada, los cuales se unen a células endoteliales y monocitos, pudiendo desempeñar un papel importante en las complicaciones de la $\mathrm{DM}^{7}$.

Sin embargo, si DM y enfermedad periodontal son procesos inflamatorios crónicos, la consecuente liberación de citoquinas proinflamatorias de una de ellas podría influir el curso de la otra y viceversa. Actualmente, se discute la posibilidad 
que la enfermedad periodontal influya en el control de los niveles glicémicos; el objetivo de esta revisión de la literatura, verificar a través de una búsqueda en el portal PubMed (www.pubmed. gov), de ensayos clínicos, con los términos "type 2 diabetes" y "periodontal disease", limitado a estudios con "humanos" y "ensayos clínicos", con por lo menos 3 meses de seguimiento, si la enfermedad periodontal ejerce influencia sobre la condición metabólica de la DM-2.

\section{Revisión de la literatura}

La búsqueda fue realizada en septiembre de 2008 y entregó un total de dieciséis artículos. Fueron excluidos dos de ellos que se referían a la influencia de la DM sobre la enfermedad periodontal; dos artículos que no fueron considerados ensayos clínicos; y un artículo que no tuvo un seguimiento mínimo. Quedando un total de once artículos (Tabla 1).

\section{Terapia periodontal mecánica sobre el control de la DM-2}

Kiran et $\mathrm{al}^{8}$, en 2005, investigaron el efecto de la salud periodontal sobre el control sistémico de la DM-2, en un estudio donde reunieron 44 pacientes con DM-2 divididos en dos grupos: uno recibió tratamiento periodontal (TP), y el otro grupo (Control) que no recibió tratamiento. Fueron evaluados parámetros clínicos periodontales, niveles de glicemia, hemoglobina glicosilada ( $\mathrm{HbA1c}$ ), colesterol total, triglicéridos, y microalbuminuria, al inicio y después de 3 meses. Los resultados demostraron mejorías en el cuadro clínico periodontal y disminución significativa en los niveles de HbAlc en el grupo TP, mientras el grupo Control demostró un pequeño aumento de HbAlc. Los autores concluyeron que el tratamiento periodontal está asociado al control glicémico de la DM-2.

En 2006, Faria-Almeida et al ${ }^{9}$, compararon la respuesta al tratamiento periodontal de pacientes con y sin DM-2, distribuyendo 10 pacientes para cada grupo. De los pacientes DM-2 fueron obtenidos los niveles de Hb1Ac y glicemia. Estos indicadores fueron registrados al inicio, a los 3 y 6 meses. Los resultados demostraron mejorías clínicas del estado periodontal en los dos grupos. Los niveles de $\mathrm{HbAlc}$ fueron estadísticamente diferentes entre el inicio y los 3 meses, asi como entre el inicio y los 6 meses. La glicemia en ayuno, a pesar de haber disminuido, no alcanzó diferencias estadísticas. Los autores concluyeron que los dos grupos demostraron buenas respuestas al tratamiento periodontal, y que pacientes DM-2 demostraron disminución en los niveles de $\mathrm{HbAlc}$ a los 3 y 6 meses.

Jones et $\mathrm{al}^{10}$, en 2007, evaluaron el efecto de la asistencia periodontal sobre el control glicémico de pacientes adultos, sobre los 65 años de edad con DM no compensada. Ciento sesenta y cinco pacientes con DM fueron distribuidos en dos grupos: terapia periodontal inmediata (TPI) o asistencia y cuidados usuales (ACU), es decir, aquellos que el sujeto realizaría aun cuando no estuviera participando de la investigación. Los parámetros evaluados fueron $\mathrm{HbAlc}$ y el porcentaje de participantes con disminución en los niveles de $\mathrm{HbAlc}$. Los resultados demostraron que sujetos del grupo ACU tenían 2 veces más probabilidad de presentar elevación en los niveles de glicemia basal y menos probabilidad de reducir su HbAlc.

En 2008, Madden et $\mathrm{al}^{11}$, evaluaron el efecto de mínima intervención periodontal (MI) o terapia frecuente (TF) sobre el control metabólico de la DM. Reunieron 50 pacientes con DM-2 descompensados y con gingivitis o periodontitis. Se realizó orientación de higiene bucal (OHB), destartraje y pulido radicular superficial (DPR) al inicio. El grupo MI volvió a los 6 meses para reforzar la $\mathrm{OHB}$ y nuevo DPR. El grupo TF recibió bimestralmente OHB y DPR, totalizando 4 sesiones. Se evaluaron, parámetros clínicos periodontales y $\mathrm{HbAlc}$, que fueron obtenidos al inicio, a los 6 y a los 8 meses. Los resultados demostraron diferencias estadísticas en los parámetros clínicos a los 6 meses en relación al inicio en los dos grupos, pero no en la reducción de $\mathrm{HbAl}$ c en cualquier grupo o tiempo. Las medidas de reducción de HbAlc fueron de 0,58 (TF) y 0,64 (MI) unidades. Se concluyó que una modesta reducción en los niveles de HbAlc fue obtenida, con una tendencia de mejores resultados, para el protocolo de TF.

\section{Terapia periodontal mecánica con antibioticoterapia sistémica sobre el control de la DM-2}

En 1997, Grossi et al ${ }^{12}$, evaluaron el efecto de la terapia periodontal sobre el control metabólico de DM en 113 pacientes con enfermedad periodontal y DM-2, los cuales fueron ubicados en 5 grupos de tratamiento: 1) DPR asociada a doxici- 
Tabla 1- Representación y síntesis de los artículos revisados en el estudio

\begin{tabular}{|c|c|c|c|c|}
\hline Autor, Año & Población & Intervención / Comparación & Tiempo & Resultados \\
\hline Kiran, 2005 & 44 con DM-2 & $\begin{array}{l}\text { Terapia periodontal } \\
\text { 1) NT } \\
\text { 2) DPR }\end{array}$ & 3 meses & $\begin{array}{l}\text { Disminución significante } \\
\text { HbA1c en el grupo } 2\end{array}$ \\
\hline Faria-Almeida, 2006 & $\begin{array}{l}20 \text { con perio- } \\
\text { dontitis, con } \\
y \sin \mathrm{DM}-2\end{array}$ & $\begin{array}{l}\text { Terapia periodontal } \\
\text { 1) Con DM-2 } \\
\text { 2) } \operatorname{Sin} \mathrm{DM}-2\end{array}$ & 6 meses & $\begin{array}{l}\text { Dos grupos responde- } \\
\text { rán bien al tratamiento } \\
\text { periodontal } \\
\text { Reducción significativa de } \\
\text { HbA1c en el grupo } 1\end{array}$ \\
\hline Jones, 2007 & $\begin{array}{l}165 \text { jubilados } \\
\text { con DM-2 }\end{array}$ & $\begin{array}{l}\text { Terapia periodontal } \\
\text { 1) DPR } \\
\text { 2) Cuidados de rutina }\end{array}$ & 4 meses & $\begin{array}{l}\text { Sin diferencias significati- } \\
\text { vas entre los grupos en la } \\
\text { reducción de HbA1c }\end{array}$ \\
\hline Madden, 2008 & $\begin{array}{l}42 \text { con EPy } \\
\text { DM-2 }\end{array}$ & $\begin{array}{l}\text { Terapia periodontal } \\
\text { 1) Mínima Intervención } \\
\text { 2) Terapia Frecuente }\end{array}$ & 6 meses & $\begin{array}{l}\text { Modesta reducción } \mathrm{HbA} 1 \mathrm{c} \\
\text { en los dos grupos }\end{array}$ \\
\hline Grossi, 1997 & $\begin{array}{l}113 \text { America- } \\
\text { nos con DP y } \\
\text { DM-2 }\end{array}$ & $\begin{array}{l}\text { Terapia periodontal + antibiótico } \\
\text { sistémico } \\
\text { 1) } D P R+D S \\
\text { 2) } D P R+C H X+D S \\
\text { 3) } D P R+P V P I+D S \\
\text { 4) } D O R+C H X+P \\
\text { 5) } D P R+\text { agua + P }\end{array}$ & 6 meses & $\begin{array}{l}\text { Reducción significativa de } \\
\text { HbA1c en los grupos } 1, \\
2 \text { y } 3 \text {. }\end{array}$ \\
\hline Rodrigues, 2003 & $\begin{array}{l}30 \text { con } \\
\text { periodontitis } \\
\text { y DM-2 }\end{array}$ & $\begin{array}{l}\text { Terapia periodontal + antibiótico } \\
\text { sistémico } \\
\text { 1) DPR } \\
\text { 2) DPR + AMOX-AC }\end{array}$ & 3 meses & $\begin{array}{l}\text { Reducción significativa de } \\
\mathrm{HbA} 1 \mathrm{c} \text { en el grupo } 1\end{array}$ \\
\hline Promsudthi, 2005 & $\begin{array}{l}52 \text { con } \\
\text { periodontitis } \\
\text { y DM-2 }\end{array}$ & $\begin{array}{l}\text { Terapia periodontal + antibiótico } \\
\text { sistémico } \\
\text { 1) NT } \\
\text { 2) DPR + DS }\end{array}$ & 3 meses & $\begin{array}{l}\text { No encontró diferencia } \\
\text { en los niveles de } \mathrm{HbA} 1 \mathrm{c} \\
\text { intra o intergrupos a los } \\
3 \text { meses }\end{array}$ \\
\hline Yun, 2007 & $\begin{array}{l}46 \text { con } \\
\text { periodontitis } \\
\text { y DM-2 }\end{array}$ & $\begin{array}{l}\text { Terapia periodontal + antibiótico } \\
\text { sistémico } \\
\text { 1) DS } \\
\text { 2) DPR + DS }\end{array}$ & 4 meses & $\begin{array}{l}\text { Disminución significati- } \\
\text { va de } \mathrm{HbA} 1 \mathrm{c} \text { en los dos } \\
\text { grupos }\end{array}$ \\
\hline O'Connell, 2008 & $\begin{array}{l}30 \text { con } \\
\text { periodontitis } \\
\text { y DM-2 }\end{array}$ & $\begin{array}{l}\text { Terapia periodontal + antibiótico } \\
\text { sistémico } \\
\text { 1) DPR + P } \\
\text { 2) DPR + DS }\end{array}$ & 3 meses & $\begin{array}{l}\text { Disminución HbA1c sin di- } \\
\text { ferencia en los dos grupos }\end{array}$ \\
\hline Rocha, 2001 & $\begin{array}{l}40 \text { con } \\
\text { periodontitis } \\
\text { y DM-2 }\end{array}$ & $\begin{array}{l}\text { Terapia periodontal + alendronato } \\
\text { 1) } D P R+P \\
\text { 2) } D P R+\text { alendronato }\end{array}$ & 6 meses & $\begin{array}{l}\text { No hubo diferencias en la } \\
\text { reducción de HbA1c en los } \\
\text { dos grupos }\end{array}$ \\
\hline Al-Mubarak, 2002 & $\begin{array}{l}52 \text { con perio- } \\
\text { dontitis cróni- } \\
\text { ca y DM-1 o } \\
\text { DM-2 }\end{array}$ & $\begin{array}{l}\text { Terapia periodontal + irrigación } \\
\text { subgingival } \\
\text { 1) DPR } \\
\text { 2) DPR + irrigación subgingival } \\
\text { con agua }\end{array}$ & 3 meses & $\begin{array}{l}\text { No encontró diferencia } \\
\text { estadística entre los gru- } \\
\text { pos en la disminución de } \\
\text { HbA1c }\end{array}$ \\
\hline
\end{tabular}

Abreviaciones: DPR- destartraje y pulido radicular; P- placebo; DS- doxiciclina sistémica; NA- no se aplica; NT- ningún tratamiento; EP- enfermedad periodontal; DM- diabetes mellitus; CHX- clorhexidina; PVPI- yodopovidona. 
clina sistémica $100 \mathrm{mg}$ por 2 semanas $(\mathrm{DPR}+\mathrm{D})$; 2) DPR asociada al uso tópico de clorhexidina $0,12 \%$ y doxiciclina sistémica $100 \mathrm{mg}$ por 2 semanas (DPR+DC); 3) DPR asociado al uso tópico de povidona yodada y doxiciclina sistémica 100 mg por 2 semanas (DPR+DI); 4) DPR asociado al uso tópico de clorhexidina $0,12 \%$ y placebo (DPR+PC); 5) DPR asociado al uso tópico de agua y placebo (Control). Se evaluaron parámetros clínicos periodontales, detección de Porphyromonas gingivalis $(P g)$ en la placa subgingival, así como la glicemia en ayuno y $\mathrm{HbA} 1 \mathrm{c}$ al inicio, a los 3 y 6 meses. Los resultados mostraron que los tres grupos que hicieron uso de doxiciclina sistémica presentaron la mayor reducción de sacos periodontales, menores niveles de detección de $\mathrm{Pg}$, y reducción significativa en los niveles de HbAlc a los 3 meses. Los autores concluyeron que el tratamiento efectivo de la infección periodontal y la reducción de la inflamación periodontal están asociados a una reducción en los niveles de HbAlc.

Después, en 2003, Rodrigues et $\mathrm{al}^{13}$, monitorearon el efecto de la terapia periodontal en el control glicémico en pacientes con DM-2. Reunieron 30 pacientes con DM-2 y periodontitis. Los pacientes fueron divididos en dos grupos: un grupo recibió DPR, mientras el otro recibió DPR asociado a amoxicilina con ácido clavulánico por 14 días (DPR+A). Se observaron parámetros clínicos periodontales, niveles de glicemia en ayuno, así como HbA1c al inicio y a los 3 meses. Los resultados demostraron mejorías en el cuadro clínico periodontal y en los niveles de HbA1c para los dos grupos. Sin embargo, sólo el grupo DPR obtuvo una diferencia estadísticamente significativa, con una reducción considerable en los niveles de HbA1c. Concluyeron que el tratamiento periodontal mejoro el control glicémico en pacientes con DM-2 en ambos grupos. Sin embargo, sólo el grupo DPR consiguió diferencias estadísticamente significativas respecto de la reducción de HbA1c.

En 2005, Promsudthi et al ${ }^{14}$, evaluaron el efecto de la terapia periodontal sobre pacientes con DM2. Reunieron 52 pacientes con DM-2, que fueron distribuidos en dos grupos: terapia periodontal asociada a doxiciclina sistémica (TD) y ningún tratamiento (Control). Fueron obtenidos los parámetros clínicos periodontales, la glicemia en ayuno, asi como los niveles de HbAlc al inicio y después de 3 meses. Los resultados demostraron mejorías estadísticamente significativas en el cuadro clíni- co periodontal del grupo TD, mientras el grupo control tuvo aumento de pérdida de inserción. Los niveles de HbAlc y glicemia no demostraron diferencias significativas entre el inicio y 3 meses, así como no hubo diferencia entre los grupos a los 3 meses. Concluyeron que hay mejorías clínicas significativas en las condiciones periodontales después de la terapia asociada a los antibióticos, siendo la progresión de pérdida de inserción rápida en pacientes no tratados.

Yun et $\mathrm{al}^{15}$, en 2007, evaluaron el efecto de la terapia periodontal en parámetros clínicos y control glicémico de pacientes DM-2 con periodontitis. Cuarenta y seis pacientes fueron divididos en dos grupos: Sólo con Doxiciclina sistémica (D) o asociada a DPR (DPR-D). Los parámetros fueron evaluados al inicio y después de 4 meses. Los autores encontraron una reducción significativa de los niveles de HbA1c en los dos grupos. A los 4 meses el grupo DPR-D demostró mejores resultados en relación a los parámetros clínicos periodontales que el grupo D. Los autores concluyeron que la terapia periodontal puede ser efectiva en la mantención de la salud bucal y puede reducir los niveles de $\mathrm{HbAlc}$ en pacientes DM-2 con periodontitis.

En 2008, O'Connell et al $^{16}$, evaluaron los efectos de la terapia periodontal sobre los niveles de HbA1c y citoquinas y quimiocinas circulantes. Investigaron en 30 pacientes con DM-2 descompensados y periodontitis. Los pacientes fueron distribuidos aleatoriamente en un grupo para DPR asociado a placebo (DPR-P) y un DPR con doxiciclina $100 \mathrm{mg} /$ día (DPR-D). La medicación sistémica fue prescrita por dos semanas, iniciando un día antes del tratamiento periodontal. Se investigaron los parámetros clínicos, HbAlc, glicemia en ayuno y marcadores inflamatorios. Los datos fueron obtenidos al inicio y a los 3 meses después de la terapia periodontal. Después de 3 meses de la terapia, el grupo DPR-P tuvo una disminución media de $0,9 \%$ versus $1,5 \%$ del grupo DPR-D en los niveles de HbAlc. La disminución después de 3 meses de la terapia en el grupo DPR-D es significativa, pero la comparación entre los dos grupos no demostró diferencias estadísticas. Hubo reducción significativa de citoquinas y quimiocinas circulantes a los 3 meses, pero sin diferencia estadística entre los grupos. Los autores concluyeron que el DPR puede influir en las condiciones sistémicas de pacientes DM-2, pero que no hubo diferencias estadísticas significativas en relación al uso adjunto de doxiciclina sistémica. 
Terapia periodontal mecánica con otras terapias complementarias sobre el control de la DM-2

En 2001, Rocha et $\mathrm{al}^{17}$, estudiaron el efecto del alendronato en la prevención de la perdida ósea en pacientes con enfermedad periodontal y DM2. Cuarenta pacientes fueron distribuidos en 2 grupos: tratamiento con alendronato $10 \mathrm{mg}$ /día (A) y placebo (P) por 6 meses. Todos los pacientes incluidos en el estudio recibieron DPR en la evaluación inicial. Se evaluó la distancia entre la cresta ósea alveolar y la unión esmalte-cemento, un marcador bioquímico de reabsorción ósea (N-telopeptídeo de la orina/NTx), parámetros clínicos periodontales, creatinina, glicemia en ayuno y $\mathrm{HbAlc}$ al inicio y después de 6 meses. El grupo A demostró superioridad en relación a disminuir NTx en 6 meses, mejorando los parámetros clínicos periodontales y disminuyendo la distancia entre la cresta ósea alveolar y la unión cemento-esmalte. Sin embargo, los dos tratamientos no demostraron diferencias en relación a los niveles de $\mathrm{HbA1c}$. Se concluyó que el alendronato indujo superiores mejorías clínicas en la altura de la cresta ósea comparado con la terapia de control en pacientes con DM-2.

Al-Mubarak et a ${ }^{18}$, en 2002, evaluaron la eficacia de la terapia periodontal asociada a irrigación subgingival en 52 pacientes diabéticos (DM-1 y DM-2) que fueron divididos en dos grupos: DPR y DPR asociado a irrigación subgingival casera dos veces al día con cantidades correspondientes a una reserva de agua y durante el tiempo del estudio (DPR+I). Se evaluaron parámetros clínicos periodontales, citocinas circulantes, generación de especies reactivas de oxígeno (ERO), $\mathrm{HbAlc}$ al inicio, a las 6 y 12 semanas después del tratamiento. El grupo DPR+I demostró diferencias estadísticas comparado con el grupo DPR, mejorando en relación a los parámetros clínicos periodontales y ERO a las 12 semanas. Además, sólo el grupo DPR+I demostró reducción de IL-1 en 6 semanas y $\mathrm{PGE}_{2}$ en 6 y 12 semanas. Los autores concluyeron que la irrigación subgingival es un complemento importante al DPR en el restablecimiento de la salud periodontal en pacientes diabéticos.

\section{Discusión}

Parece bastante evidente la influencia de ciertas condiciones sistémicas como la DM en la prevalencia y severidad de la enfermedad periodontal ${ }^{2-4}$, pero de la enfermedad periodontal en el control de la DM aún es motivo de discusión. La posibilidad de que la terapia periodontal favorezca el control de los niveles glicéricos está siendo centro de muchos estudios, como los revisados anteriormente.

A pesar de la controversia en relación a la representatividad de las diferencias presentadas entre los grupos evaluados, es importante resaltar que si bien los estudios no demuestran diferencias significativas en sus resultados, se observa una tendencia de disminución en los niveles de $\mathrm{HbAlc}$ en los grupos que recibieron intervención periodontal. En este sentido es importante distinguir entre diferencia estadística y diferencia clínica. La variación en el rango entre "salud" y "enfermedad" es mínima, y puede no ser sensible al examen estadístico. Una reducción de $0,6 \%$ en los niveles de HbAlc puede no ser estadísticamente significativa, pero es clínicamente deseada ${ }^{11}$. Una reducción de sólo 1\% en los niveles de $\mathrm{HbAlc}$ demostró reducir los infartos al miocardio en $14 \%{ }^{19}$, mientras un aumento de $1 \%$ en estos valores eleva a $25 \%$ la chance de complicaciones sistémicas ${ }^{20}$.

Otro punto a ser considerado en los artículos, se refiere al tiempo del estudio, el número de pacientes evaluados y la metodología empleada. El análisis de glicemia en ayuno, por ejemplo, está sujeto a grandes oscilaciones y por esto no es considerado un buen indicador de control glicémico. La glucosa se une irreversiblemente a la hemoglobina y cuanto mayor sea la concentración de glucosa sanguínea, mayor será la cantidad de uniones entre la glucosa y las moléculas de hemoglobina. La hemoglobina glicosilada refleja el nivel medio de glucosa de los 2 a 3 meses previos al examen. Por lo tanto, idealmente los estudios que evalúan este indicador, deberían tener una duración de por lo menos 3 meses para lograr detectar las diferencias entre el inicio y el término del tratamiento ${ }^{22}$. Cinco de los estudios revisados evaluaron niveles de HbAlc utilizando 3 meses de seguimiento ${ }^{8,13-14,16,18}$. De estos, se hizo una comparación entre un grupo no tratado contra otro grupo que recibió terapia periodontal en apenas un estudio, sin encontrar diferencias estadísticas entre ambos ${ }^{14}$. La literatura apunta a que períodos mayores de seguimiento son necesarios para garantizar la confiabilidad de estos estudios.

A pesar de que pocos estudios han evaluado su efecto y los pocos realizados han encontrado poca o ninguna diferencia estadística, la asociación de 
antibióticos a DPR puede ser un diferencial en el control metabólico del paciente. O'Connell et al ${ }^{16}$, atribuyeron el mayor resultado de la asociación de doxiciclina con DPR cuando es comparado a DPR sin asociación, a los efectos adicionales, antimicrobianos y antiinflamatorios del antibiótico. La doxiciclina, además de ser de amplio espectro y efectivo contra la mayor cantidad de patógenos periodontales, es un potente modulador de la respuesta del hospedero en individuos DM-2, inhibiendo la acción de metaloproteasas, la glicosilación no enzimática de proteínas extracelulares y posiblemente de la hemoglobina ${ }^{12}$. Sin embargo, por la posibilidad de efectos adversos más severos y prolongados, se sugiere que la medicación sistémica con doxiciclina sea sustituida por el tratamiento local con la misma, terapia que también es efectiva en disminuir los niveles sanguíneos de $\mathrm{HbAlc}^{22}$. Pero Rodrigues et $\mathrm{al}^{13}$, fallaron en demostrar una asociación positiva entre DPR y amoxicilina asociada al ácido clavulánico en el control metabólico de $\mathrm{HbA1c}$. Se cree que la administración de amoxicilina en este caso, fue perjudicada por la posible presencia de microorganismos no sensibles en el saco periodontal, especialmente por la presencia de complejos microbianos en el biofilm subgingival.

Variaciones entre los estudios también deberían ser eliminadas, estandarizando una metodología para obtener una conclusión satisfactoria del tema. En la única revisión sistemática ${ }^{21}$ se encontró una reducción no significativa de $0,66 \%$ en los niveles de $\mathrm{HbA1c}$ posterior a la terapia periodontal en pacientes con DM-2, aumentando este valor a $0,71 \%$ cuando antibióticos fueron administrados conjuntamente. Se concluyó que el tratamiento periodontal no afecta el control glicémico en pacientes diabéticos. Sin embargo, los autores criticaban la falta de estandarización de los estudios en relación a dieta, índice de masa corporal y otros factores de riesgo para DM-2, tamaño de la muestra, tiempo, duración y niveles de $\mathrm{HbA} 1 \mathrm{c}$ al inicio.

Los reales efectos de la terapia periodontal pueden ser enmascarados por efectos psicológicos consecuentes de la participación de un individuo en un estudio clínico, el llamado efecto Hawthorne. Este efecto ocurre cuando una variable comportamental es afectada por un factor no directamente relacionado a ella y que muchas veces no puede ser controlado ${ }^{23}$.
Así, Kinane y Bouchard ${ }^{24}$ afirmaron que no se puede concluir si el tratamiento periodontal consigue mejorías en el control glicémico. Sin embargo, recomiendan que el clínico esté informado sobre la relación bidireccional entre DM, enfermedad periodontal y sus ramificaciones clínicas, para diagnóstico y tratamiento.

Después de estas consideraciones, se concluye por el análisis de la literatura revisada, que la enfermedad periodontal ejerce una influencia sobre la condición metabólica de la DM-2. Sin embargo, para la comprensión adecuada de esta interacción son necesarios nuevos estudios considerando nuevas variables, grupos experimentales mayores y un tiempo de seguimiento más prolongado.

\section{Referencias}

1. The American Academy of Periodontology. Glossary of periodontal terms. $4^{\text {th }}$ ed. The American Academy of Periodontology: Chicago, 2001.

2. Mealey BL, Oates TW. Diabetes mellitus and periodontal diseases. J Periodontol 2006; 77: 1289-303.

3. Mealey BL, Ocampo GL. Diabetes mellitus and periodontal disease. Periodontol 2000 2007; 44: 127-53.

4. Preshaw PM, Foster N, Taylor JJ. Cross-susceptibility between periodontal disease and type 2 diabetes mellitus: an immunobiological perspective. Periodontol 2000 2007; 45: 138-57.

5. Novak MJ, Potter RM, Blodgett J, Ebersole JL. Periodontal disease in Hispanic Americans with type 2 diabetes. J Periodontol 2008; 79: 629-36.

6. Southerland JH, Taylor GW, Moss K, Beck JD, Offenbacher S. Commonality in chronic inflammatory diseases: periodontitis, diabetes, and coronary artery disease. Periodontol 2000 2006; 40: 130-43.

7. Hudson BI, Bucciarelli LG, Wendt T, Sakaguchi T, Lalla E, Qu W, et al. Blockade of receptor for advanced glycation endproducts: a new target for therapeutic intervention in diabetic complications and inflammatory disorders. Archives of Biochemistry and Biophysics 2003; 419: 80-8.

8. Kiran M, Arpak N, Ünsal E, Erdogan MF. The effect of improved periodontal health on metabolic control in type 2 diabetes mellitus. J Clin Periodontol 2005; 32: 266-72.

9. Faria-Almeida R, Navarro A, Bascones A. Clinical and metabolic changes after conventional treatment of type 2 diabetic patients with chronic periodontitis. J Periodontol 2006; 77: 591-8. 
10. Jones JA, Miller DR, Wehler CJ, Rich SE, Krall-Kaye EA, Mccoy LC, et al. Does periodontal care improve glycemic control? The Department of Veterans Affairs Dental Diabetes Study. J Clin Periodontol 2007; 34: 46-52.

11. Madden TE, Herriges B, Boyd L, Laughlin G, Chiodo GT, Rosenstein DI. Alterations in HbAlc following minimal or enhanced non-surgical, non-antibiotic treatment of gingivitis or mild periodontitis in type 2 diabetic patients: a pilot trial. J Contemp Dent Pract 2008; 9: 9-16.

12. Grossi SG, Skrepcinski FB, Decaro T, Robertson DC, Ho AW, Dunford RG, et al. Treatment of periodontal disease in diabetics reduces glycated hemoglobin. J Periodontol 1997; 68: 713-9.

13. Rodrigues DC, Taba-Jr M, Novaes-Jr AB, Souza SLS, Grisi MFM. Effect of non-surgical periodontal therapy on glycemic control in patients with type 2 diabetes mellitus. J Periodontol 2003; 74: 1361-7.

14. Promsudthi A, Pimapansri S, Deerochanawong C, Kanchanavasita W. The effect of periodontal therapy on uncontrolled type 2 diabetes mellitus in older subjects. Oral Diseases 2005; 11: 293-8.

15. Yun F, Firkova EI, Jun-Oi L, Xun H. Effect of non-surgical periodontal therapy on patients with type 2 diabetes mellitus. Folia Med (Plovdiv) 2007; 49: 32-6.

16. O'Connell PAA, Taba-Jr M, Nomizo A, Freitas MCF, Suaid FA, Uyemura AS, et al. Effects of periodontal therapy on glycemic control and inflammatory markers. J Periodontol 2008; 79: 774-83.

17. Rocha M, Nava LE, De La Torre CV, Sánchez-Marín F, Garay-Sevilla ME, Malacara JM. Clinical and radiolo- gical improvement of periodontal disease in patients with type 2 diabetes mellitas treated with alendronate: a randomized, placebo-controlled trial. J Periodontol 2001; 72: 204-9.

18. Al-Mubarak S, Ciancio S, Aljada A, Awa H, Hamouda W, Ghanim $\mathrm{H}$, et al. Comparative evaluation of adjunctive oral irrigation in diabetics. J Clin Periodontol 2002; 29: 295-300.

19. Fisher M. Prevention of macrovascular complications. European Heart J Supplements 2003; 5 (Suppl B): B21B26.

20. Schellhase KG, Koepsell TD, Weiss NS. Glycemic control and the risk of multiple microvascular diabetic complications. Fam Med 2005; 37: 125-30.

21. Janket SJ, Wightman A, Baird AE, Van Dyke TE, Jones JA. Does periodontal treatment improve glycemic control in diabetic patients? A meta-analysis of intervention studies. J Dent Res 2005; 84: 1154-9.

22. Iwamoto $\mathrm{Y}$, Nishimura $F$, Nakagawa $M$, Sugimoto $H$, Shikata K, Makino H, et al. The effect of antimicrobial periodontal treatment on circulating tumor necrosis factor-alpha and glycated hemoglobin level in patients with type 2 diabetes. J Periodontol 2001; 72: 774-8.

23. Watts T. Periodontal treatment and glycemic control in diabetic patients: the problem of a possible Hawthorne effect. J Dent Res 2006; 85: 294-5.

24. Kinane D, Bouchard P. Periodontal diseases and health: consensus report of the sixth European workshop on Periodontology. J Clin Periodontol 2008; 35 (Suppl 8): 333-7. 